\title{
Análise vocal e laríngea na hipótese diagnóstica de nódulos e cistos
}

\author{
Vocal and laryngeal analyses in diagnostic hypotheses of \\ nodules and cysts
}

\author{
Lívia Fernandes Barata1 ${ }^{1}$, Glaucya Madazio², Mara Behlau, Osíris do Brasil ${ }^{4}$
}

\begin{abstract}
RESUMO
Objetivo: Verificar via fonação inspiratória, se parâmetros visuais e perceptivo-auditivos relacionam-se com a hipótese diagnóstica de nódulos e cistos. Métodos: Foram analisadas 21 videolaringoestroboscopias de pacientes com nódulos ( $\mathrm{n}=6$ ) e cistos ( $\mathrm{n}=15)$, média de idade de 35 anos (13-68 anos), 18 mulheres e três homens. Dados visuais e perceptivo-auditivos dos exames, avaliados na fonação expiratória e inspiratória, foram apresentados aleatoriamente, comparados e registrados em protocolo específico, por fonoaudiólogo especialista em voz, desconhecedor da hipótese diagnóstica. Resultados: Nos nódulos, a vogal sustentada evidenciou maior ocorrência de desvios vocais $(66,7 \%)$, do que a fala encadeada (33,3\%); a vogal sustentada apresentou-se soprosa (100\%) e a fala encadeada, adaptada (66,7\%). Nos cistos, o desvio foi mais frequente na vogal sustentada $(46,7 \%)$ do que na fala encadeada (40\%); a vogal sustentada apresentou tanto componente rugoso (40\%) como soproso (33,3\%). Todos os nódulos foram simétricos em localização (100\%) e a maioria foi simétrica em tamanho, em ambas as fonações (66,7\% e $75 \%$, respectivamente). Os cistos tenderam à simetria em localização (75\%), mas não foram simétricos em tamanho (100\%). O ligamento vocal foi mais visível nos cistos $(53,3 \%$ e $80 \%)$ do que nos nódulos (33,3\% e 66,7\%), respectivamente, na fonação expiratória e inspiratória, e mais evidente durante fonação inspiratória. Quando visível nos nódulos, o ligamento vocal foi sempre bilateral (100\%). Conclusão: A fonação inspiratória revelou características diferenciais para nódulos e cistos; a análise perceptivo-auditiva ofereceu informação adicional na caracterização das lesões.
\end{abstract}

Descritores: Distúrbios da voz/diagnóstico; Cordas vocais; Laringe/patologia

\section{INTRODUÇÃO}

A manobra de fonação inspiratória, também chamada fonação inalatória ou reversa foi descrita ${ }^{(1)}$ por meio de análise radiológica da laringe. Tal manobra consiste na produção da voz durante a entrada de ar nos pulmões, mecanismo inverso do habitual, pois o ar é sonorizado durante a inspiração; as pregas vocais se apõem e o ar inspiratório passa a ser parcialmente bloqueado, provocando queda na pressão subglótica ${ }^{(2)}$.

Trabalho realizado no Curso de Especialização em Voz pelo Centro de Estudos da Voz - CEV - São Paulo (SP), Brasil, como monografia de conclusão de curso.

(1) Pós-graduanda (Mestrado) em Ciências na Área de Oncologia pelo Hospital AC Camargo - São Paulo (SP), Brasil.

(2) Doutora, Professora do Curso de Especialização do Centro de Estudos da Voz - CEV - São Paulo (SP), Brasil.

(3) Doutora, Diretora do Centro de Estudos da Voz - CEV - São Paulo (SP), Brasil.

(4) Doutor, Professor do Curso de Especialização do Centro de Estudos da Voz - CEV - São Paulo (SP), Brasil.

Endereço para correspondência: Lívia Fernandes Barata. R. Tamandaré, 246/203, Liberdade, São Paulo (SP), Brasil, CEP: 01525-000. E-mail: liviabarata@gmail.com

Recebido em: 9/10/2008; Aceito em: 21/1/2010
A diferença de pressão propicia o relaxamento do vestíbulo laríngeo com visualização dos ventrículos, seios piriformes e região posterior da laringe ${ }^{(1,3,4)}$. É considerado um recurso para a especificação das lesões de massa, auxiliando na identificação de cistos de pregas vocais e na determinação da integridade da lâmina própria ${ }^{(4,5)}$. É considerada técnica facilitadora( ${ }^{(6)} \mathrm{e}$ tem sido utilizada pelos fonoaudiólogos para a desativação da fonação vestibular, aproximação das pregas vocais e estimulação da onda de mucosa $a^{(2,6,7)}$.

O nódulo vocal como entidade é uma lesão benigna que ocorre em consequência do excesso de abusos vocais por atrito constante, geralmente na região do terço médio das pregas vocais; a microscopia mostra espessamento epitelial e da membrana basal. Apresenta-se à laringoscopia como lesão nodular bilateral com tamanhos variados e geralmente simétricos ${ }^{(8,9)}$.

O cisto é considerado uma alteração estrutural mínima de cobertura das pregas vocais, localizado profundamente no interior da prega vocal, em geral na camada superficial da lâmina própria, com ou sem aderência ao ligamento vocal; pode ser uni ou bilateral ${ }^{(8,10)}$.

A literatura sugere que muitas das lesões de pregas vocais ocorrem como resultado de traumas repetidos, que podem 
produzir lesões bilaterais ou também reações contralaterais na presença de uma alteração estrutural mínima específica ${ }^{(11-13)}$.

Lesões de massa como nódulos e cistos podem ser de difícil distinção visual e, além de apresentarem características vocais semelhantes, o diagnóstico diferencial destas lesões exige análise perceptivo-auditiva e visual acuradas ${ }^{(8,11,14)}$. A fonação inspiratória pode ser utilizada para auxiliar na melhor definição das lesões de massa. Contudo, o diagnóstico de uma lesão pode ser complexo e só é obtido após a conclusão do tratamento ${ }^{(8,10)}$. Quando não é possível realizar um diagnóstico preciso, apesar de terem sido utilizados todos os recursos da semiologia, a conduta é realizar a fonoterapia diagnóstica, com o objetivo de auxiliar na definição do diagnóstico da lesão ${ }^{(2)}$. Neste contexto, insere-se a importância do trabalho conjunto entre fonoaudiólogo e médico para avaliação e conduta, estabelecendo contribuição diagnóstica na identificação e caracterização destas lesões.

$\mathrm{Na}$ análise perceptivo-visual da laringe, por meio da estroboscopia, durante a fonação inspiratória, o ligamento vocal geralmente aparece bem configurado e totalmente evidente nos nódulos, com disposição irregular ou não evidente nos cistos. Portanto, a possibilidade de se visualizar o ligamento vocal fornece dados sobre a presença e localização da lesão ${ }^{(4)}$. Os nódulos revelam-se livres, destacando-se do ligamento vocal, que se torna bem delineado. Ao contrário, os cistos geralmente mostram-se aderidos ou semi-aderidos, permanecendo evidentes, em destaque na borda ou face superior das pregas vocais, enquanto os nódulos são tracionados inferiormente, tornando-se menos salientes ${ }^{(8)}$.

Nódulos, por se tratarem de lesões mais superficiais, não possuem comprometimento evidente da onda mucosa e vibram com o resto da mesma, destacados do ligamento vocal; já nos cistos intracordais, que apresentam uma íntima relação com o ligamento vocal, o prejuízo da onda mucosa é maior, observando-se uma lesão rígida, com possível área silente ou vibração muito reduzida nos tecidos circunvizinhos ${ }^{(8)}$. Nas pregas vocais, a lesão cística costuma localizar-se no plano subepitelial, entre epitélio e ligamento/músculo vocal, na maioria das vezes no terço médio da prega vocal ${ }^{(15-17)}$.

Nos nódulos iniciais e edematosos, a qualidade vocal resultante pode ser apenas levemente rouca ou soprosa, chegando a ser adaptada em alguns casos. Nos nódulos mais antigos e rígidos, pode ocorrer abafamento de todo o processo de oscilação da prega vocal, com maior aperiodicidade de vibração, maior perturbação de frequência e maior rouquidão, podendo haver presença de aspereza. As características tensionais podem estar presentes, especialmente quando se observa o envolvimento da musculatura paralaríngea, com desvio da frequência fundamental em direção aos sons agudos. Já os cistos podem ser assintomáticos, levemente sintomáticos, ou com alterações vocais caracterizadas por voz rouca, devido à irregularidade dos ciclos glóticos e, grave, devido ao aumento de massa na prega vocal ${ }^{(8)}$. Quanto melhor a vibração das pregas vocais, melhor a qualidade vocal ${ }^{(18)}$. À análise perceptivo-auditiva da voz, de modo geral, a rouquidão e a soprosidade são os principais sinais indicativos de presença de lesão de massa em pregas vocais como os nódulos e cistos, sendo difícil diferenciar ambas as lesões apenas pela qualidade vocal. A rouquidão correlaciona-se ao grau de irregularidade de vibração das pregas vocais, enquanto a soprosidade referese ao fechamento glótico incompleto. Ambos, nódulo e cisto, apresentam irregularidade de vibração e fechamento glótico incompleto $^{(8,19)}$.

Desta forma, o objetivo do presente trabalho foi verificar se parâmetros visuais e perceptivo-auditivos, analisados dos trechos de fonação expiratória e inspiratória durante o exame laríngeo, relacionam-se com a hipótese médica diagnóstica de nódulos e cistos.

\section{MÉTODOS}

Este estudo é de caráter retrospectivo e foi aprovado pelo Comitê de Ética do Centro de Estudos da Voz (CEV) - São Paulo, sob o número 2614/06. O material analisado corresponde a 21 exames videolaringoestroboscópicos de pacientes adultos, realizados em clínica otorrinolaringológica por médico especializado em avaliação de laringe, selecionados a partir do ano de 2005. Os exames apresentavam boa certeza de acurácia da hipótese diagnóstica ${ }^{(2)}$ de cisto $(n=15)$ e nódulo vocal $(n=6)$, à videoestrobolaringoscopia. Os critérios de inclusão foram: hipótese diagnóstica otorrinolaringológica de cistos ou nódulos de pregas vocais e realização adequada da fonação inspiratória. Foram excluídos os exames com qualidade visual ou de áudio comprometidas ou aqueles em que não se obteve visualização total das estruturas da laringe em ambas as modalidades de fonação: expiratória e inspiratória.

Dos 26 exames iniciais, quatro foram excluídos pela impossibilidade de visualização total da laringe e um por baixa qualidade de áudio. Desta forma, o estudo foi realizado com 21 indivíduos, média de idade de 35 anos, entre as faixas etárias de 13 a 68 anos, de ambos os sexos (18 mulheres e três homens).

O material de fala utilizado foi a fonação expiratória, com emissão da vogal "é" sustentada e fala encadeada (meses do ano), e a fonação inspiratória, com a produção da vogal "i" prolongada, durante uma inspiração bucal. Ambas as modalidades de fonação foram gravadas durante videolaringoestroboscopia, realizada por um único médico otorrinolaringologista.

Antes de o paciente submeter-se ao exame de laringe, as vozes foram captadas por um microfone da marca Multilaser®, preso à região do tórax, com distância de aproximadamente 15 $\mathrm{cm}$ da boca do paciente, acoplado ao equipamento para exame otorrinolaringológico.

A videolaringoestroboscopia foi realizada com telescópio rígido marca Machida ${ }^{\circledR}$, modelo LCY-32. $70^{\circ}$, microcâmera marca Toshiba ${ }^{\circledR}$, DVD marca Philips ${ }^{\circledR}$, fonte de luz XE 1800, gravado em CD RW marca Sony®. Os pacientes foram anestesiados com xilocaína spray a 10\%, anestesia tópica.

A opção pelo telescópio rígido para realização dos exames teve a intenção de se obter máxima qualidade das imagens com melhor visualização das lesões para um melhor diagnóstico.

As gravações, que incluíam voz e imagem laríngea, foram editadas e organizadas de forma aleatória. Posteriormente, foram analisadas por três fonoaudiólogos especialistas em voz. Como opção estatística, foram consideradas as respostas do juiz com maior índice de confiabilidade inter e intra- 
avaliadores. Os avaliadores não estavam cientes da hipótese diagnóstica médica estabelecida.

A análise do material foi realizada por meio de análise perceptivo-auditiva e visual de gravação em $\mathrm{CD}$ de cada paciente, nas emissões em fonação expiratória e inspiratória. $\mathrm{O}$ juiz recebeu o $\mathrm{CD}$ com as amostras gravadas e editadas, e as avaliou em computador próprio, de forma independente. As respostas foram registradas em protocolo específico (Anexo 1).

Para a análise perceptivo-auditiva da qualidade vocal, foram avaliados dois momentos, ou seja, durante a emissão sustentada da vogal "é", e durante a fala encadeada, ambos gravados com microfone supra citado, acoplado ao equipamento de exame laríngeo. Os parâmetros selecionados para avaliação foram: 1) tipo de voz predominante, classificada em adaptada, rugosa, soprosa, tensa ou outra; 2) grau global de desvio, classificado em ausente, leve, moderado ou severo.

Para a análise perceptivo-visual das estruturas da laringe foram considerados: 1. presença de lesão nas pregas vocais, classificada em ausente, não-avaliável, presente unilateral ou bilateral (simetria em localização e tamanho); 2. constrição supraglótica, classificada em ausente, não avaliável e presente, além do tipo apresentado (mediana, ântero-posterior, global); 3. visualização do ligamento vocal: não visível e visível, quando se observava a linha do ligamento indicada por uma faixa mais escura, completa ou incompleta (unilateral ou bilateral); 4. fenda fonatória: ausente (quando a coaptação entre as pregas vocais era completa), não avaliável (quando a constrição supraglótica interferia na visualização das pregas vocais), presente: dupla, triangular médio posterior, ampulheta, triangular posterior, fusiforme anterior, irregular ${ }^{(8)}$.

Os resultados foram apresentados de forma descritiva, com média simples e porcentagem. As medidas de tendência central (média e mediana) e de variabilidade (variação mínima/máxima) e desvio padrão foram utilizadas para descrever as variáveis numéricas e, para as categóricas, a distribuição de frequências. Trata-se de uma pesquisa clínico-qualitativa, de natureza descritiva, logo não se propõe a generalizações estatísticas, mas o estabelecimento de indicadores analíticos.

\section{RESULTADOS}

Na Tabela 1, observa-se que os nódulos mostraram mais desvios vocais na vogal sustentada do que na fala encadeada, apresentando qualidade vocal soprosa na vogal sustentada e adaptada na fala encadeada. Nos cistos, a alteração da voz aparece tanto na vogal sustentada como na fala encadeada; na vogal sustentada observa-se componente rugoso ou soproso. Diferente dos nódulos, os cistos mantiveram a disfonia na fala encadeada, apresentando rugosidade.

Todos os nódulos foram simétricos em localização e tendem a ser simétricos em tamanho (Tabela 2). Os cistos, quando bilaterais, durante fonação expiratória, apresentam tendência à simetria em localização e assimetria em tamanho; na fonação inspiratória, tendem à simetria em localização e são assimétricos em tamanho.

A constrição supraglótica manteve-se a mesma, em ambas as fonações, tanto nos cistos como nos nódulos (Tabela 2).

A visualização do ligamento vocal é evidente na execução da fonação inspiratória para ambas as lesões. Quando visível, nos cistos, evidencia-se bilateral em 62,5\% dos casos à fonação expiratória e, 66,7\%, à fonação inspiratória. Nos nódulos, o ligamento vocal é visível bilateralmente em todos os casos (Tabela 2).

Em todos os casos de nódulos, a coaptação glótica apresenta-se incompleta, sendo que a maioria é classificada como dupla. Grande parte dos cistos apresenta fenda fonatória, duplas ou irregulares, um caso apresenta coaptação completa e outro coaptação não avaliável, em função da falta de visualização total das pregas vocais por constrição glótica (Tabela 2).

\section{DISCUSSÃO}

A visualização direta das estruturas da laringe e do padrão vibratório das pregas vocais é fundamental tanto para o diagnóstico como para o tratamento apropriado das disfonias. O processo de decisão clínica e a interpretação da imagem laríngea envolvem julgamentos perceptivos da imagem das estruturas da laringe e seus movimentos vibratórios ${ }^{(21)}$.

Tabela 1. Análise perceptivo-auditiva da vogal sustentada "é" e da fala encadeada

\begin{tabular}{|c|c|c|c|c|c|c|c|c|}
\hline \multirow[b]{3}{*}{ Parâmetro } & \multicolumn{4}{|c|}{ Cisto } & \multicolumn{4}{|c|}{ Nódulo } \\
\hline & \multicolumn{2}{|c|}{ Vogal } & \multicolumn{2}{|c|}{ Fala } & \multicolumn{2}{|c|}{ Vogal } & \multicolumn{2}{|c|}{ Fala } \\
\hline & $\mathrm{N}$ & $\%$ & $\mathrm{~N}$ & $\%$ & $\mathrm{~N}$ & $\%$ & $\mathrm{~N}$ & $\%$ \\
\hline \multicolumn{9}{|c|}{ Tipo de voz predominante } \\
\hline Adaptada & 4 & 26,7 & 4 & 26,7 & 0 & 0 & 4 & 66,7 \\
\hline Rugosa & 6 & 40 & 10 & 66,7 & 0 & 0 & 1 & 16,7 \\
\hline Soprosa & 5 & 33,3 & 1 & 6,7 & 6 & 100 & 1 & 16,7 \\
\hline Tensa & 0 & 0 & 0 & 0 & 0 & 0 & 0 & 0 \\
\hline Outra & 0 & 0 & 0 & 0 & 0 & 0 & 0 & 0 \\
\hline \multicolumn{9}{|c|}{ Grau global de desvio } \\
\hline Ausente & 4 & 26,7 & 4 & 26,7 & 0 & 0 & 4 & 66,7 \\
\hline Leve & 7 & 46,7 & 6 & 40 & 4 & 66,7 & 0 & 0 \\
\hline Moderado & 4 & 26,7 & 5 & 33,3 & 2 & 33,3 & 2 & 33,3 \\
\hline Severo & 0 & 0 & 0 & 0 & 0 & 0 & 0 & 0 \\
\hline
\end{tabular}


Tabela 2. Análise perceptivo-visual das estruturas da laringe na fonação expiratória e inspiratória

\begin{tabular}{|c|c|c|c|c|c|c|c|c|}
\hline \multirow[t]{3}{*}{ Parâmetro } & \multicolumn{4}{|c|}{ Cisto } & \multicolumn{4}{|c|}{ Nódulo } \\
\hline & \multicolumn{2}{|c|}{ Fonação exp } & \multicolumn{2}{|c|}{ Fonação insp } & \multicolumn{2}{|c|}{ Fonação exp } & \multicolumn{2}{|c|}{ Fonação insp } \\
\hline & $\mathrm{N}$ & $\%$ & $\mathrm{~N}$ & $\%$ & $\mathrm{~N}$ & $\%$ & $\mathrm{~N}$ & $\%$ \\
\hline \multicolumn{9}{|l|}{ Lesão nas PPVV } \\
\hline Ausente & 2 & 13,3 & 6 & 40 & 0 & 0 & 2 & 33,3 \\
\hline Presente unilateral & 4 & 26,7 & 5 & 33,3 & 0 & 0 & 0 & 0 \\
\hline \multicolumn{9}{|l|}{ simetria em localização } \\
\hline não & 3 & 33,3 & 1 & 25 & 0 & 0 & 0 & 0 \\
\hline $\operatorname{sim}$ & 6 & 66,7 & 3 & 75 & 6 & 100 & 4 & 100 \\
\hline \multicolumn{9}{|l|}{ simetria em tamanho } \\
\hline não & 6 & 66,7 & 4 & 100 & 2 & 33,3 & 1 & 25 \\
\hline $\operatorname{sim}$ & 3 & 33,3 & 0 & 0 & 4 & 66,7 & 3 & 75 \\
\hline \multicolumn{9}{|l|}{ Constrição supraglótica } \\
\hline Ausente & 13 & 86,7 & 13 & 86,7 & 3 & 50 & 3 & 50 \\
\hline Presente & 2 & 13,3 & 2 & 13,3 & 3 & 50 & 3 & 50 \\
\hline mediana & 0 & 0 & 0 & 0 & 0 & 0 & 1 & 33,3 \\
\hline antero-posterior & 1 & 50 & 1 & 50 & 2 & 66,7 & 1 & 33,3 \\
\hline global & 1 & 50 & 1 & 50 & 1 & 33,3 & 1 & 33,3 \\
\hline \multicolumn{9}{|l|}{ Ligamento vocal } \\
\hline Não visível & 7 & 46,7 & 3 & 20 & 4 & 66,7 & 2 & 33,3 \\
\hline Visível & 8 & 53,3 & 12 & 80 & 2 & 33,3 & 4 & 66,7 \\
\hline unilateral & 3 & 37,5 & 4 & 33,3 & 0 & 0 & 0 & 0 \\
\hline bilateral & 5 & 62,5 & 8 & 66,7 & 2 & 100 & 4 & 100 \\
\hline \multicolumn{9}{|l|}{ Fenda fonatória } \\
\hline Ausente & 1 & 6,7 & & & 0 & 0 & & \\
\hline Não avaliável & 1 & 6,7 & & & 0 & 0 & & \\
\hline Presente & 13 & 86,7 & & & 6 & 100 & & \\
\hline dupla & 5 & 38,5 & & & 4 & 66,7 & & \\
\hline triangular médio-posterior & 0 & 0 & & & 1 & 16,7 & & \\
\hline ampulheta & 1 & 7,7 & & & 0 & 0 & & \\
\hline triangular posterior & 1 & 7,7 & & & 0 & 0 & & \\
\hline fusiforme anterior & 2 & 15,4 & & & 0 & 0 & & \\
\hline irregular & 4 & 30,8 & & & 1 & 16,7 & & \\
\hline
\end{tabular}

Legenda: Fonação exp = fonação expiratória; Fonação insp = fonação inspiratória; PPVV = pregas vocais

A fonação inspiratória durante o exame laringológico revelou características diferenciais para as hipóteses diagnósticas médicas de nódulos e cistos, acrescentando dados à avaliação feita na fonação expiratória e inspiratória, já que, durante este tipo de diagnóstico, estas lesões são frequentemente confundidas. Nos casos de lesões bilaterais existem dúvidas entre o lado da lesão propriamente dito e o lado da reação contralateral. Alterações na mucosa circunvizinha às lesões podem dificultar a visualização e, muitas vezes, o diagnóstico acaba sendo atribuído como lesão de massa uni ou bilateral a esclarecer.

Neste estudo, os nódulos apresentaram maior número de desvios na vogal sustentada do que na fala encadeada. Isso ocorre, provavelmente, porque durante a fala os desvios são reduzidos, devido à influência da articulação, ressonância, ritmo e velocidade de fala, seleção de ajustes musculares e psicodinâmica vocal ${ }^{(18)}$. A qualidade vocal predominante nessas lesões foi a soprosa, provavelmente pela protrusão lingual necessária durante o exame e pelo baixo número de casos analisados nesta pesquisa. Nos cistos, o desvio apareceu tanto na vogal sustentada como na fala, com componente rugoso ou soproso na vogal sustentada, e rugoso na fala encadeada, traduzida por irregularidade de vibração de mucosa ${ }^{(8)}$. Neste estudo, não apareceu o componente tensão para os nódulos, conforme descrito na literatura.

De uma forma geral, a fonação inspiratória foi manobra útil na identificação e diferenciação entre nódulos vocais e cistos. O estudo auxiliou na observação do tamanho e simetria da lesão, que pode auxiliar no diagnóstico diferencial. À fonação inspiratória houve uma tendência dos nódulos serem simétricos em tamanho e, no entanto, todos os cistos foram assimétricos 
em tamanho; ou seja, se a lesão é simétrica em localização e simétrica em tamanho, a tendência do diagnóstico é ser de nódulo; se for simétrica em localização e assimétrica em tamanho, a tendência é ser de cisto. Os nódulos revelaram-se mais flexíveis, destacando-se do ligamento vocal, que se torna bem delineado durante a fonação inspiratória. Ao contrário, os cistos mostraram-se aderidos ou semi-aderidos, permanecendo evidentes, em destaque na borda ou face superior das pregas vocais, enquanto os nódulos são tracionados inferiormente, indicando que a visualização do ligamento vocal depende da ocorrência da expansão da lâmina própria. O ligamento vocal foi sempre visível bilateralmente durante a fonação inspiratória nos nódulos. Em alguns casos de cisto a visualização foi unilateral.

Embora a técnica de fonação inspiratória seja indicada para reduzir a constrição supraglótica ${ }^{(6)}$, neste estudo, não houve modificação das constrições entre as duas modalidades de fonação. À expiração e à inspiração, cistos e nódulos apresentaram constrição supraglótica. É interessante ressaltar que a constrição mais comumente descrita em casos de nódulo, que é a mediana, ocorreu em apenas um caso.

Evidentemente, a presença de uma lesão de massa pode impedir uma coaptação adequada das pregas vocais ${ }^{(8)}$ e interferir negativamente na função vocal ${ }^{(21,22)}$. Todos os nódulos apresentaram coaptação incompleta e grande parte dos cistos também. A fenda mais comum nos nódulos foi dupla, e nos cistos tanto a dupla como a irregular. Logo, fica difícil a diferenciação das lesões pelo tipo de fenda. A coaptação incompleta, característica dessas lesões, produz alterações importantes e modifica a relação existente entre o sinal e o ruído da voz, percebido do ponto de vista auditivo e passível de ser mensurado por inúmeras estratégias acústicas ${ }^{(23)}$.

O diagnóstico diferencial definitivo entre nódulos e cistos não pôde ser realizado apenas com os dados do exame, mas as informações adicionais sobre a lesão oferecem maior sustentação à hipótese diagnóstica, bem como as características perceptivo-auditivas analisadas, em cada caso.

Houveram certas limitações no estudo pela dificuldade em se fazer uma correlação entre nódulos e cistos pela ausência de diagnóstico definitivo das lesões. Portanto, é interessante a realização de uma pesquisa para o acompanhamento de casos, na microlaringoscopia exploratória e posterior exame histopatológico da lesão, além da análise acústica e comportamental e terapia diagnóstica ${ }^{(24)}$, para melhor comparação das informações concedidas no exame de laringe, já que a presença de limitações é inerente a todos os métodos de análise.

\section{CONCLUSÃO}

O estudo da análise auditiva da voz e visual da laringe na hipótese diagnóstica de nódulo vocal e cisto permitiu concluir que houve relação entre os parâmetros visuais e perceptivoauditivos selecionados, analisados dos trechos de fonação expiratória e inspiratória durante o exame laríngeo, com a hipótese médica diagnóstica de nódulo e cisto.

\section{AGRADECIMENTOS}

Às fonoaudiólogas Irene de Pedro Netto, Mônica Bretas e Fabiana Zambom pela valorosa contribuição científica e disponibilidade durante a realização deste.

\begin{abstract}
Purpose: To verify, using inspiratory phonation, whether visual and auditory-perceptual parameters correlate with the diagnostic hypothesis of nodules and cysts. Methods: Twenty one videolaryngostroboscopies of patients with suspected nodules ( $\mathrm{n}=6$ ) and cysts $(n=15)$ were analyzed. Subjects were 18 women and three men, with mean age of 35 years (13-68 years). Visual and auditory data, obtained from expiratory and inspiratory phonation, were randomly presented, compared and registered in a specific protocol by a trained speech-language pathologist, to whom diagnostic hypotheses were omitted. Results: In nodule cases, sustained vowel showed higher occurrence of vocal deviation $(66.7 \%)$, when compared to speech $(33.3 \%)$; sustained vowel had a breathy quality (100\%) and speech was adapted $(66.7 \%)$. In cyst cases, deviation was also more frequent during sustained vowel (46.7\%) than during speech (40\%); sustained vowel presented both roughness (40\%) and breathiness (33.3\%). All nodules were symmetric in location (100\%), and most were also symmetric in size, in both types of phonation (66.7\% and $75 \%$ respectively). Cysts tended to be symmetric in location $(75 \%)$, but asymmetric in size $(100 \%)$. The vocal ligament was more visible in cysts $(53.3 \%$ and $80 \%)$ than in nodules $(33.3 \%$ and $66.7 \%$ ), in expiratory and inspiratory phonation respectively, and this structure was more evident during inspiratory phonation. When visible in nodules, the vocal ligament was bilateral (100\%). Conclusion: Inspiratory phonation revealed differential characteristics for nodules and cysts diagnoses; the auditory-perceptual evaluation added information to characterize both lesions.
\end{abstract}

Keywords: Voice disorders/diagnosis; Vocal folds; Larynx/pathology 


\section{REFERÊNCIAS}

1. Powers WE, Holtz S, Ogura J. Contrast examination of the larynx and pharynx. Inspiratory phonation. Am J Roentgenol Radium Ther Nucl Med. 1964;92:40-2.

2. Behlau M, Madazio G, Feijó D, Azevedo R, Gielow I, Rehder MI. Aperfeiçoamento vocal e tratamento fonoaudiológico das disfonias. In: Behlau M, editor. Voz o livro do especialista. Vol. 2. Rio de Janeiro: Revinter; 2005. p.409-528.

3. Lehmann QH. Reverse phonation: a new maneuver for examining the larynx. Radiology. 1965;84:215-22.

4. Lopes MV, Behlau M, Brasil OOC, Andrade D. A utilização da fonação inspiratória na caracterização das lesões benignas da laringe. Rev Bras Otorrinolaringol. 2000;66(5):512-8.

5. Finger LS, Cielo CA. Aspectos fisiológicos e clínicos da técnica fonoterapêutica de fonação reversa. Rev Bras Otorrinolaringol. 2007;73(2):271-7.

6. Boone DR, McFarlane SC. The voice and voice therapy. 4th ed. New Jersey: Prentice-Hall; 1988. Chapter 6: Voice therapy for special problems; p. 156- 74.

7. Nemetz MA, Pontes PAL, Vieira VP, Yazaki RK. Configuracão das pregas vestibulares à fonacão em adultos com e sem disfonia. Rev Bras Otorrinolaringol. 2005;71(1):6-12.

8. Behlau M, Madazio G, Feijó D, Pontes P. Avaliação de voz. In: Behlau M, editor. Voz o livro do especialista. Vol. 1. Rio de Janeiro: Revinter; 2001. p.85-120.

9. De Biase N, Pontes P, Vieira VP, De Biase S. O modo de coaptação glótica em crianças no diagnóstico diferencial de alteração estrutural mínima. Rev Bras Otorrinolaringol. 2004;70(4):457-62.

10. Pontes P, Gonçalves MI, Behlau M. Vocal fold cover minor structural alterations. Phonoscope. 1999;2(4):175-85.

11. Rosen CA, Lombard LE, Murry T. Acoustic, aerodynamic and videostroboscopic features of bilateral vocal fold lesions. Ann Otol Rhinol Laryngol. 2000;109(9):823-8.

12. D'Avila JS, Sennes LU, Tsuji DH. Estudo comparativo da microvascularização das pregas vocais humanas acometidas por cisto e reação nodular contra-lateral sob endoscopia rígida. Rev Bras Otorrinolaringol. 2003;69(2):166-73.

13. Altman KW. Vocal fold masses. Otolaryngol Clin North Am. 2007;40(5):1091-108, viii.

14. Pastana SG, Gomes E, Castro L. Conduta fonoaudiológica e avaliação estroboscópica no diagnóstico diferencial do cisto. Rev CEFAC. 2007;9(3):397-403.

15. Sato K, Hirano M. Age-related changes of elastics fibers in the superficial layer of the lamina propria of vocal folds. Ann Otol Rhinol Laryngol. 1997;106(1):44-8.

16. Pontes PAL, De Biase NG, Behlau M. Vascular characteristics of the vocal fold cover in control larynges and larynges with benign lesions. Phonoscope. 1999;2(3):129-35.

17. Colton RH, Casper JK. Compreendendo os problemas de voz: uma perspectiva fisiológica ao diagnóstico e ao tratamento. Porto Alegre: Artes Médicas; 1996.

18. Rosen CA, Anderson D, Murry T. Evaluating hoarseness: keeping your patient's voice healthy. Am Fam Physician. 1998;57(11):2775-82.

19. Behlau M, Gasparini G. Classification Manual for Voice Disorders-I -CMVD-I. Rev Soc Bras Fonoaudiol. 2007;12(1):72-5.

20. Patel RR. Visual perceptions in laryngeal imaging. Perspect Voice Voice Disord. 2007;17:7-10.

21. Omori K, Slavit DH, Kacker A, Blaugrund SM. Influence of size and etiology of glottal gap in glottic incompetence dysphonia. Laryngoscope. 1998;108(4 Pt 1):514-8.

22. Leeper WR, Fung K, Beaudin PG, Doyle PC. Voice-related quality of life in patients with benign vocal fold lesions. J Otolaryngol Head Neck Surg. 2008;37(3):423-9.

23. Santos IR, Dell'Acqua RM, Cunto MR, Pereira JC. Parâmetros acústicos da voz relacionados à soprosidade. Rev Soc Bras Fonoaudiol. 2005;10(1):53-9.

24. Ulis JM, Yanagisawa E. What's new in differential diagnosis and treatment of hoarseness? Curr Opin Otolaryngol Head Neck Surg. 2009;17(3):209-15.

Anexo 1. Protocolo utilizado para avaliação perceptivo-auditiva e perceptivo-visual dos exames de viodeolaringoscopia

\begin{tabular}{|c|c|c|}
\hline \multicolumn{3}{|c|}{ Análise perceptivo-auditiva: vogal e } \\
\hline Tipo de voz predominante & \multicolumn{2}{|c|}{ ( ) adaptada ( ) rugosa ( ) soprosa } \\
\hline Grau global de desvio & \multicolumn{2}{|c|}{$($ ) ausente ( ) leve $\quad($ ) moderado ( ) severo } \\
\hline \multicolumn{3}{|c|}{ Análise preceptivo-auditiva: fala encadeada/meses do ano } \\
\hline Tipo de voz predominante & \multicolumn{2}{|c|}{ ( ) adaptada ( ) rugosa ( ) soprosa } \\
\hline Grau global de desvio & \multicolumn{2}{|c|}{ ( ) ausente ( ) leve ( ) moderado ( ) severo } \\
\hline \multicolumn{3}{|c|}{ Análise perceptivo-visual fonação expiratória } \\
\hline Lesão nas PPVV & ( ) unilateral & $\begin{array}{l}\text { ( ) bilateral: } \\
\begin{array}{lll}\text { simetria em localização ( ) não } & \text { ( ) sim } \\
\text { simetria em tamanho } & \text { ( ) não } & \text { ( ) sim }\end{array}\end{array}$ \\
\hline Fenda fonatória & \multicolumn{2}{|c|}{ ( ) não ( ) sim tipo: } \\
\hline Constrição supraglótica (na situação de conforto) & \multicolumn{2}{|c|}{ ( ) não ( ) sim ( ) mediana ( ) ântero-posterior ( ) global } \\
\hline Visualização do ligamento vocal & \multicolumn{2}{|c|}{ ( ) não ( ) sim ( ) unilateral ( ) bilateral } \\
\hline \multicolumn{3}{|c|}{ Análise perceptivo-visual fonação inspiratória } \\
\hline Lesão nas PPVV & ( ) unilateral & $\begin{array}{l}\text { ( ) bilateral: } \\
\begin{array}{lll}\text { simetria em localização ( ) não } & \text { ( ) sim } \\
\text { simetria em tamanho } & \text { ( ) não } & \text { ( ) sim }\end{array}\end{array}$ \\
\hline Constrição supraglótica (na situação de conforto) & ( ) não ( ) sim & ( ) mediana ( ) ântero-posterior ( ) global \\
\hline Visualização do ligamento vocal & ( ) não ( ) sim & ( ) unilateral ( ) bilateral \\
\hline
\end{tabular}

Legenda: PPVV = pregas vocais 